\title{
Non-Differentiable Solution of Nonlinear Biological Population Model on Cantor Sets
}

\author{
Djelloul Ziane $^{1}$, Mountassir Hamdi Cherif ${ }^{1, *(\mathbb{D}}$, Dumitru Baleanu ${ }^{2,3}$ (1) and Kacem Belghaba ${ }^{1}$ \\ 1 Laboratory of Mathematics and Its Applications (LAMAP), University of Oran1 Ahmed Ben Bella, \\ 31000 Oran, Algeria; djeloulz@yahoo.com (D.Z.); belghaba@yahoo.fr (K.B.) \\ 2 Department of Mathematics, Cankaya University, 6690 Ankara, Turkey; dumitru@cankaya.edu.tr \\ 3 Institute of Space Sciences, 077125 Magurele-Bucharest, Romania \\ * Correspondence: mountassir27@yahoo.fr; Tel.: +213-775136242
}

Received: 14 November 2019; Accepted: 30 January 2020; Published: 9 February 2020

\begin{abstract}
The main objective of this study is to apply the local fractional homotopy analysis method (LFHAM) to obtain the non-differentiable solution of two nonlinear partial differential equations of the biological population model on Cantor sets. The derivative operator are taken in the local fractional sense. Two examples have been presented showing the effectiveness of this method in solving this model on Cantor sets.
\end{abstract}

Keywords: local fractional homotopy analysis method; nonlinear biological population model Local fractional derivative; non-differentiable solution

\section{Introduction}

Partial differential equations are more important than others, due to their multiple scientific uses, where they are involved in the study of some phenomena related to human life, organisms and the universe, such as engineering phenomena, mechanical phenomena, chemical phenomena and biological phenomena. Also, among the basic tools for analyzing physical phenomena, physical phenomena, etc., we find fractional and local fractional partial differential equations ([1-8]). Given the importance of these equations, the knowledge of their solutions is the other more important, because it enables us to study the phenomenon associated with these equations ([9-13]).

In this field there are many researchers interested in developing several methods that enable us to solve this kind of equations, among them, for example, are the Adomian decomposition method [14], homotopy perturbation method [15], variational iteration method [16], DJ-iteration method [17] and homotopy analysis method [18] and others. All this is to have an ideal method to solve all kinds of ordinary differential equations as well as all kinds of partial differential equations .

The homotopy analysis method, which was developed in 1992 by Liao Shijun ([18-21]), and has been used extensively to solve partial or ordinary differential equations or differential systems of integer order or fractional order ([22-27]). Lately, this method has been extended by S. Maitama and W. Zhao [28] to solve non-differentiable problems on Cantor sets. The objective of our paper is based on this method, where we will apply it, to solve two nonlinear partial differential equations for biological population models on Cantor sets. Note that, these models has been solved and discussed in $[29,30]$ with Caputo fractional derivative and conformable derivative operator respectively.

\section{Basic Definitions}

We will present the basic concepts of fractional local calculus, and in particular the local fractional derivative, local fractional integral, and some important results of this operator. 


\subsection{Local Fractional Derivative}

Definition 1. Setting $g(\zeta) \in C_{\sigma}(a, b)$, the local fractional derivative of $g(\zeta)$ of order $\sigma$ at $\zeta=\zeta_{0}$ is given by $([31,32])$

$$
g^{(\sigma)}(\zeta)=\left.\frac{d^{\sigma} g}{d \zeta^{\sigma}}\right|_{\zeta=\zeta_{0}}=\lim _{\zeta \rightarrow \zeta_{0}} \frac{\Delta^{\sigma}\left(g(\zeta)-g\left(\zeta_{0}\right)\right)}{\left(\zeta-\zeta_{0}\right)^{\sigma}}
$$

where

$$
\Delta^{\sigma}\left(g(\zeta)-g\left(\zeta_{0}\right)\right) \cong \Gamma(1+\sigma)\left[\left(g(\zeta)-g\left(\zeta_{0}\right)\right)\right]
$$

and $C_{\sigma}(a, b)$, designates the class of functions called local fractional continuous on the interval $(a, b)$.

The high-order local fractional derivative of $g(\zeta)$ is

$$
g^{(n \sigma)}(\zeta)=\overbrace{D_{\zeta}^{(\sigma)} \cdots D_{\zeta}^{(\sigma)} g(\zeta)}^{n \text { times }} .
$$

\subsection{Local Fractional Integral}

Definition 2. The local fractional integral of $g(\zeta)$ of order $\sigma$ in $[a, b]$ is defined as $([31,32])$

$$
\begin{aligned}
{ }_{a} I_{b}^{(\sigma)} g(\zeta) & =\frac{1}{\Gamma(1+\sigma)} \int_{a}^{b} g(\zeta)(d \zeta)^{\sigma} \\
& =\frac{1}{\Gamma(1+\sigma)} \lim _{\Delta \zeta \rightarrow 0} \sum_{j=0}^{N-1} f\left(\zeta_{j}\right)\left(\Delta \zeta_{j}\right)^{\sigma},
\end{aligned}
$$

where $\Delta \zeta_{j}=\zeta_{j+1}-\zeta_{j}, \Delta \zeta=\max \left\{\Delta \zeta_{0}, \Delta \zeta_{1}, \Delta \zeta_{2}, \cdots\right\}$ and $\left[\zeta_{j}, \zeta_{j+1}\right], \zeta_{0}=a, \zeta_{N}=b$, is a partition of $[a, b]$.

\subsection{Some Properties of the Local Fractional Operators}

The local fractional operators fulfill some fundamental equations. In particular, starting from the Mittag-Leffler function we have the following

$$
\begin{gathered}
E_{\sigma}\left(\zeta^{\sigma}\right)=\sum_{m=0}^{+\infty} \frac{\zeta^{m \sigma}}{\Gamma(1+m \sigma)}, \quad 0<\sigma \leq 1, \\
\sin _{\sigma}\left(\zeta^{\sigma}\right)=\sum_{m=0}^{+\infty}(-1)^{m} \frac{\zeta^{(2 m+1) \sigma}}{\Gamma(1+(2 m+1) \sigma)}, \quad 0<\sigma \leq 1, \\
\cos _{\sigma}\left(\zeta^{\sigma}\right)=\sum_{m=0}^{+\infty}(-1)^{m} \frac{\zeta^{2 m \sigma}}{\Gamma(1+2 m \sigma)}, \quad 0<\sigma \leq 1 .
\end{gathered}
$$

By using the local fractional derivative (1) and the definitions (3) it can be easily shown that $([31,32])$

$$
\begin{aligned}
\frac{d^{\sigma}}{d \zeta^{\sigma}} \frac{\zeta^{m \sigma}}{\Gamma(1+m \sigma)}= & \frac{\zeta^{(m-1) \sigma}}{\Gamma(1+(m-1) \sigma)}, \\
\frac{d^{\sigma}}{d \zeta^{\sigma}} E_{\sigma}\left(\zeta^{\sigma}\right) & =E_{\sigma}\left(\zeta^{\sigma}\right) \\
\frac{d^{\sigma}}{d \zeta^{\sigma}} \sin _{\sigma}\left(\zeta^{\sigma}\right) & =\cos _{\sigma}\left(\zeta^{\sigma}\right), \\
\frac{d^{\sigma}}{d \zeta^{\sigma}} \cos _{\sigma}\left(\zeta^{\sigma}\right) & =-\sin _{\sigma}\left(\zeta^{\sigma}\right),
\end{aligned}
$$




$$
{ }_{0} I_{\zeta}^{(\sigma)} \frac{\zeta^{m \sigma}}{\Gamma(1+m \sigma)}=\frac{\zeta^{(m+1) \sigma}}{\Gamma(1+(m+1) \sigma)} .
$$

\section{Local Fractional Homotopy Analysis Method}

Maitama and Zhao [28] introduced the basis idea of this method, where they considered the nonlinear partial differential equation with local fractional derivative

$$
N[w(\chi, \zeta)]=0,
$$

where $N$ denote the nonlinear operator, $w(\chi, \zeta)$ is the local fractional unknown function. By using the homotopy analysis method [18], we construct a convex non-differentiable homotopy

$$
(1-q) L_{\sigma}\left[\varphi(\chi, \zeta ; q)-w_{0}(\chi, \zeta)\right]=q \hbar H(\chi, \zeta) N[\varphi(\chi, \zeta ; q)]
$$

with the embedding parameter $q \in[0,1], \hbar \neq 0$ is the nonzero convergence-control parameter, $H(\chi, \zeta) \neq 0$ is the local fractional nonzero auxiliary function, $\varphi(\chi, \zeta ; q)$ is the local fractional unknown function, and $w_{0}(\chi, \zeta)$ is an initial guess of $w(\chi, \zeta), L_{\sigma}=\frac{\partial^{\sigma}}{\partial \zeta^{\sigma}}$ denote the linear local fractional operator, such as

$$
L_{\sigma}[\varphi(\chi, \zeta)]=0 \text {, when } \varphi(\chi, \zeta)=0 .
$$

According to homotopy analysis method, when $q=0$ and $q=1$, we have

$$
\varphi(\chi, \zeta ; 0)=w_{0}(\chi, \zeta) \text { and } \varphi(\chi, \zeta ; 1)=w(\chi, \zeta)
$$

respectively. Thus as $q$ increases from 0 to 1 , the solution $\varphi(\chi, \zeta ; q)$ varies from $w_{0}(\chi, \zeta)$ to $w(\chi, \zeta)$. The expanding of $\varphi(\chi, \zeta ; q)$ with respect to $q$, is given by

$$
\varphi(\chi, \zeta ; q)=w_{0}(\chi, \zeta)+\sum_{m=1}^{+\infty} w_{m}(\chi, \zeta) q^{m}
$$

where

$$
w_{m}(\chi, \zeta)=\left[\frac{1}{m !} \frac{\partial^{m} \varphi(\chi, \zeta ; q)}{\partial q^{m}}\right]_{q=0} .
$$

At the correct selection of the auxiliary linear operator, the initial guess, and the convergence-control parameter, the (17) converges at $q=1$

$$
w(\chi, \zeta)=w_{0}(\chi, \zeta)+\sum_{m=1}^{+\infty} w_{m}(\chi, \zeta)
$$

it is a solution of (13).

The local fractional vector is given by

$$
\mathbf{w}_{m}=\left\{w_{0}(\chi, \zeta), w_{1}(\chi, \zeta), \ldots, w_{m}(\chi, \zeta)\right\}
$$

Differentiating of (14) $m$-times with respect to $q$ and then setting $q=0$ and finally dividing by $m$ ! gives

$$
L_{\sigma}\left[w_{m}(\chi, \zeta)-\varsigma_{m} w_{m-1}(\chi, \zeta)\right]=\hbar H(\chi, \zeta) \Re_{m}\left(\mathbf{w}_{m}, \chi, \zeta\right),
$$

where

$$
\Re_{m}\left(\mathbf{w}_{m}, \chi, \zeta\right)=\left[\frac{1}{(m-1) !} \frac{\partial^{m-1} N[\varphi(\chi, \zeta ; q)]}{\partial q^{m-1}}\right]_{q=0},
$$


and

$$
\varsigma_{m}= \begin{cases}0, & m \leq 1, \\ 1, & m>1 .\end{cases}
$$

By applying the local fractional integral operator to (21), we get

$$
\begin{aligned}
w_{m}(\varphi, \zeta)= & \varsigma_{m} w_{m-1}(\chi, \zeta)-\varsigma_{m} \sum_{k=0}^{m-1} w_{m-1}^{(k)}\left(\chi, 0^{+}\right) \frac{\zeta^{k \sigma}}{\Gamma(k \sigma+1)} \\
& +\hbar_{\beta} I_{\alpha}^{(\sigma)}\left[H(\chi, \zeta) \Re_{m}\left(\mathbf{w}_{m}, \chi, \zeta\right)\right] .
\end{aligned}
$$

The series solutions of $w_{m}(\chi, \zeta)$ for $m \geq 1$ are given by

$$
w(\chi, \zeta)=\sum_{m=0}^{+\infty} w_{m}(\chi, \zeta)
$$

For the convergence of the series (25), you can see [28].

\section{Applications}

In this section, we will apply the method described above which is attributed to Maitama and Zhao [28] to solve two nonlinear partial differential equations. These equations are called "biological population models" where the derivative operator are taken in the local fractional sense.

Example 1. First, we consider the local fractional nonlinear biological population model

$$
\frac{\partial^{\sigma} \mathscr{\omega}}{\partial \zeta^{\sigma}}=\frac{\partial^{2 \sigma} \mathcal{\omega}^{2}}{\partial \eta^{2 \sigma}}+\frac{\partial^{2 \sigma} \omega^{2}}{\partial \mu^{2 \sigma}}-\omega\left(1+\frac{8}{9} \omega\right)=0,0<\sigma \leq 1,
$$

subject to the IC

$$
\omega(\eta, \mu, 0)=E_{\sigma}\left(\frac{1}{3}(\eta+\mu)^{\sigma}\right)
$$

According to (26) and based on the steps of this method, we choose $\omega_{0}(\eta, \mu, \zeta)=E_{\sigma}\left(\frac{1}{3}(\eta+\mu)^{\sigma}\right)$ to be the initial guess.

We can take the linear operator as

$$
L_{\sigma}[\varphi(\eta, \mu, \zeta ; q)]=\frac{\partial^{\sigma}}{\partial \zeta^{\sigma}}[\varphi(\eta, \mu, \zeta ; q)] .
$$

The nonlinear operator is given as

$$
\begin{aligned}
N[\varphi(\eta, \mu, \zeta ; q)]= & \frac{\partial^{\sigma} \varphi(\eta, \mu, \zeta ; q)}{\partial \zeta^{\sigma}}-\frac{\partial^{2 \sigma} \varphi^{2}(\eta, \mu, \zeta ; q)}{\partial \eta^{2 \sigma}}-\frac{\partial^{2 \sigma} \varphi^{2}(\eta, \mu, \zeta ; q)}{\partial \mu^{2 \sigma}} \\
& +\varphi(\eta, \mu, \zeta ; q)\left(1+\frac{8}{9} \varphi(\eta, \mu, \zeta ; q)\right) .
\end{aligned}
$$

Based on the previous LFHAM ( LFHAM is Local Fractional Homotopy Analysis Method) steps, the zero-order deformation equation becomes

$$
(1-q) L\left[\varphi(\eta, \mu, \zeta ; q)-\omega_{0}(\eta, \mu, \zeta)\right]=q \hbar H(\eta, \mu, \zeta) N[\varphi(\eta, \mu, \zeta ; q)] .
$$

This leads to: when $q=0$ and $q=1$, we get

$$
\varphi(\eta, \mu, \zeta ; 0)=\omega_{0}(\eta, \mu, \zeta) \text {, and } \varphi(\eta, \mu, \zeta ; 1)=\omega(\eta, \mu, \zeta) .
$$


Then the $M^{\text {th }}$-order deformation equation is defined as

$$
L_{\sigma}\left[\omega_{m}(\eta, \mu, \zeta)-\varsigma_{m} \omega_{m-1}(\eta, \mu, \zeta)\right]=\hbar H(\eta, \mu, \zeta) \Re_{m}\left(\mathbf{\$}_{m-1}, \eta, \mu, \zeta\right),
$$

where

$$
\begin{aligned}
\Re_{m}\left(\mathbf{\$}_{m-1}, \eta, \mu, \zeta\right)= & \frac{\partial^{\sigma} \omega(\eta, \mu, \zeta)}{\partial \zeta^{\sigma}}-\frac{\partial^{2 \sigma} \omega^{2}(\eta, \mu, \zeta)}{\partial \eta^{2 \sigma}}-\frac{\partial^{2 \sigma} \omega^{2}(\eta, \mu, \zeta)}{\partial \mu^{2 \sigma}} \\
& +\omega(\eta, \mu, \zeta)\left(1+\frac{8}{9} \omega(\eta, \mu, \zeta)\right)
\end{aligned}
$$

and

$$
\varsigma_{m}= \begin{cases}0, & m \leq 1 \\ 1, & m>1\end{cases}
$$

Applying the local fractional integral on (32) and setting $H(\eta, \mu, \zeta)=1$, we have

$$
\begin{aligned}
\omega_{m}(\eta, \mu, \zeta)= & \varsigma_{m} \omega_{m-1}(\eta, \mu, \zeta)-\varsigma_{m} w(\eta, \mu, 0) \\
& +\hbar_{\beta} I_{\alpha}^{(\sigma)}\left[\begin{array}{c}
\omega(\eta, \mu, \zeta)\left(1+\frac{8}{9} \omega(\eta, \mu, \zeta)\right) \\
-\frac{\partial^{2 \sigma} \omega^{2}(\eta, \mu, \zeta)}{\partial \eta^{2 \sigma}}-\frac{\partial^{2 \sigma} \omega^{2}(\eta, \mu, \zeta)}{\partial \mu^{2 \sigma}}
\end{array}\right] .
\end{aligned}
$$

According to (35), we obtain

$$
\left\{\begin{array}{c}
\omega_{1}=\hbar_{0} I_{\zeta}^{(\sigma)}\left[\omega_{0}+\frac{8}{9}\left(\omega_{0}\right)^{2}-\frac{\partial^{2 \sigma}\left(\omega_{0}\right)^{2}}{\partial \eta^{2 \sigma}}-\frac{\partial^{2 \sigma}\left(\omega_{0}\right)^{2}}{\partial \mu^{2 \sigma}}\right] \\
\omega_{m}=(1+\hbar) \omega_{m-1}+\hbar_{0} I_{\zeta}^{(\sigma)}\left[\omega_{m-1}+\frac{8}{9} \sum_{i=0}^{m-1} \omega_{i} \omega_{m-1-i}\right] \\
-\hbar_{0} I_{\zeta}^{(\sigma)}\left[\frac{\partial^{2 \sigma}}{\partial \eta^{2 \sigma}}\left(\sum_{i=0}^{m-1} \omega_{i} \omega_{m-1-i}\right)+\frac{\partial^{2 \sigma}}{\partial \mu^{2 \sigma}}\left(\sum_{i=0}^{m-1} \omega_{i} \omega_{m-1-i}\right)\right], m>1
\end{array}\right.
$$

Through the two formulas (36), the first terms of the solution the first terms of (26), are given by

$$
\begin{gathered}
\omega_{0}(\eta, \mu, \zeta)=E_{\sigma}\left(\frac{1}{3}(\eta+\mu)^{\sigma}\right), \\
\omega_{1}(\eta, \mu, \zeta)=\hbar E_{\sigma}\left(\frac{1}{3}(\eta+\mu)^{\sigma}\right) \frac{\zeta^{\sigma}}{\Gamma(1+\sigma)}, \\
\omega_{2}(\eta, \mu, \zeta)=E_{\sigma}\left(\frac{1}{3}(\eta+\mu)^{\sigma}\right)\left[\left(\hbar+\hbar^{2}\right) \frac{\zeta^{\sigma}}{\Gamma(1+\sigma)}+\hbar^{2} \frac{\zeta^{2 \sigma}}{\Gamma(1+2 \sigma)}\right], \\
\omega_{3}(\eta, \mu, \zeta)=E_{\sigma}\left(\frac{1}{3}(\eta+\mu)^{\sigma}\right)\left[\begin{array}{c}
\hbar(1+\hbar)^{2} \frac{\zeta^{\sigma}}{\Gamma(1+\sigma)}+2\left(\hbar^{2}+\hbar^{3}\right) \frac{\zeta^{2 \sigma}}{\Gamma(1+2 \sigma)} \\
+\hbar^{3} \frac{\zeta^{3 \sigma}}{\Gamma(1+3 \sigma)}
\end{array}\right],
\end{gathered}
$$

and so on. The other terms of LFHAM can be calculated in the same way. Finally, the non-differentiable solution $\omega$ of (26) in a series, is given by

$$
\omega(\eta, \mu, \zeta)=E_{\sigma}\left(\frac{1}{3}(\eta+\mu)^{\sigma}\right)\left[\begin{array}{c}
1+\hbar\left(3+3 \hbar+\hbar^{2}\right) \frac{\zeta^{\sigma}}{\Gamma(1+\sigma)} \\
+\hbar^{2}(3+2 \hbar) \frac{\zeta^{2 \sigma}}{\Gamma(1+2 \sigma)}+\hbar^{3} \frac{\zeta^{3 \sigma}}{\Gamma(1+3 \sigma)}+\cdots
\end{array}\right] .
$$

By replacing $\hbar=-1$ in (38), we get

$$
\omega(\eta, \mu, \zeta)=E_{\sigma}\left(\frac{1}{3}(\eta+\mu)^{\sigma}\right)\left[1-\frac{\zeta^{\sigma}}{\Gamma(1+\sigma)}+\frac{\zeta^{2 \sigma}}{\Gamma(1+2 \sigma)}-\frac{\zeta^{3 \sigma}}{\Gamma(1+3 \sigma)}+\cdots\right]
$$

and also

$$
\omega(\eta, \mu, \zeta)=E_{\sigma}\left(\frac{1}{3}(\eta+\mu)^{\sigma}\right) E_{\sigma}\left(-\zeta^{\sigma}\right)=E_{\sigma}\left(\frac{1}{3}(\eta+\mu)^{\sigma}-\zeta^{\sigma}\right)
$$


Note that, in the case $\sigma=1$, we obtain the same solution obtained in [33] by homotopy perturbation method.

Example 2. Next, we consider the other local fractional nonlinear biological population model

$$
\frac{\partial^{\sigma} \omega}{\partial \zeta^{\sigma}}=\frac{\partial^{2 \sigma} \omega^{2}}{\partial \eta^{2 \sigma}}+\frac{\partial^{2 \sigma} \omega^{2}}{\partial \mu^{2 \sigma}}+k^{\sigma} \omega=0,0<\sigma \leq 1,
$$

where $k$ is a constant and with the initial condition

$$
\omega(\eta, \mu, 0)=(\sqrt{\eta \mu})^{\sigma}
$$

According to (41) and based on the steps of this method, we choose $\omega_{0}(\eta, \mu, \zeta)=(\sqrt{\eta \mu})^{\sigma}$. We take the linear operators as

$$
L_{\sigma}[\varphi(\eta, \mu, \zeta ; q)]=\frac{\partial^{\sigma}}{\partial \zeta^{\sigma}}[\varphi(\eta, \mu, \zeta ; q)] .
$$

The nonlinear operator is given as

$$
\begin{aligned}
N[\varphi(\eta, \mu, \zeta ; q)]= & \frac{\partial^{\sigma} \varphi(\eta, \mu, \zeta ; q)}{\partial \zeta^{\sigma}}-\frac{\partial^{2 \sigma} \varphi^{2}(\eta, \mu, \zeta ; q)}{\partial \eta^{2 \sigma}} \\
& -\frac{\partial^{2 \sigma} \varphi^{2}(\eta, \mu, \zeta ; q)}{\partial \mu^{2 \sigma}}-k^{\sigma} \varphi(\eta, \mu, \zeta ; q) .
\end{aligned}
$$

Based on the previous LFHAM steps, the zero-order deformation equation becomes

$$
(1-q) L\left[\varphi(\eta, \mu, \zeta ; q)-\omega_{0}(\eta, \mu, \zeta)\right]=q \hbar H(\eta, \mu, \zeta) N[\varphi(\eta, \mu, \zeta ; q)] .
$$

This leads to: when $q=0$ and $q=1$, we get

$$
\varphi(\eta, \mu, \zeta ; 0)=\omega_{0}(\eta, \mu, \zeta), \text { and } \varphi(\eta, \mu, \zeta ; 1)=\omega(\eta, \mu, \zeta)
$$

Then the $M^{\text {th }}$-order deformation equation is defined as follows

$$
L_{\sigma}\left[\omega_{m}(\eta, \mu, \zeta)-\varsigma_{m} \omega_{m-1}(\eta, \mu, \zeta)\right]=\hbar H(\eta, \mu, \zeta) \Re_{m}\left(\mathbf{\$}_{m-1}, \eta, \mu, \zeta\right),
$$

where

$$
\begin{aligned}
\Re_{m}\left(\mathbf{\$}_{m-1}, \eta, \mu, \zeta\right)= & \frac{\partial^{\sigma} \omega(\eta, \mu, \zeta)}{\partial \zeta^{\sigma}}-\frac{\partial^{2 \sigma \omega 2}(\eta, \mu, \zeta)}{\partial \eta^{2 \sigma}} \\
& -\frac{\partial^{2 \sigma \omega 2}(\eta, \mu, \zeta)}{\partial \mu^{2 \sigma}}-k^{\sigma} \omega(\eta, \mu, \zeta)
\end{aligned}
$$

and

$$
\varsigma_{m}= \begin{cases}0, & m \leq 1 \\ 1, & m>1\end{cases}
$$

Applying the local fractional integral on (47) and setting $H(\eta, \mu, \zeta)=1$, we obtain

$$
\begin{aligned}
\omega_{m}(\eta, \mu, \zeta)= & \varsigma_{m} \omega_{m-1}(\eta, \mu, \zeta)-\varsigma_{m} \omega(\eta, \zeta, 0) \\
& -\hbar_{\beta} I_{\alpha}^{(\sigma)}\left[\frac{\partial^{2 \sigma} \omega^{2}(\eta, \mu, \zeta)}{\partial \eta^{2 \sigma}}+\frac{\partial^{2 \sigma} \omega^{2}(\eta, \mu, \zeta)}{\partial \mu^{2 \sigma}}+k^{\sigma} \omega(\eta, \mu, \zeta)\right] .
\end{aligned}
$$


According to the Equation (50), we obtain

$$
\left\{\begin{array}{c}
\omega_{1}(\eta, \mu, \zeta)=-\hbar_{0} I_{\zeta}^{(\sigma)}\left[\frac{\partial^{2 \sigma}\left(\omega_{0}\right)^{2}}{\partial \eta^{2 \sigma}}+\frac{\partial^{2 \sigma}\left(\omega_{0}\right)^{2}}{\partial \mu^{2 \sigma}}+k^{\sigma} \omega_{0}\right] \\
\omega_{m}(\eta, \mu, \zeta)=(1+\hbar) \omega_{m-1}-\hbar_{0} I_{\zeta}^{(\sigma)}\left[k^{\sigma} \omega_{m-1}\right] \\
-\hbar_{0} I_{\zeta}^{(\sigma)}\left[\frac{\partial^{2 \sigma}}{\partial \eta^{2 \sigma}}\left(\sum_{i=0}^{m-1} \omega_{i} \omega_{m-1-i}\right)+\frac{\partial^{2 \sigma}}{\partial \mu^{2 \sigma}}\left(\sum_{i=0}^{m-1} \aleph_{i} \aleph_{m-1-i}\right)\right], m>1 .
\end{array}\right.
$$

Through to the formulas (51), the first terms of local fractional homotopy analysis method of (41), are given by

$$
\begin{aligned}
& \omega_{0}(\eta, \mu, \zeta)=(\sqrt{\eta \mu})^{\sigma} \\
& \omega_{1}(\eta, \mu, \zeta)=-\hbar(\sqrt{\eta \mu})^{\sigma} \frac{(k \zeta)^{\sigma}}{\Gamma(1+\sigma)^{\prime}}, \\
& \omega_{2}(\eta, \mu, \zeta)=(\sqrt{\eta \mu})^{\sigma}\left[(-\hbar)(1+\hbar) \frac{(k \zeta)^{\sigma}}{\Gamma(1+\sigma)}+\hbar^{2} \frac{(k \zeta)^{2 \sigma}}{\Gamma(1+2 \sigma)}\right], \\
& \omega_{3}(\eta, \mu, \zeta)=(\sqrt{\eta \mu})^{\sigma}\left[\begin{array}{c}
(-\hbar)(1+\hbar)^{2} \frac{(k \zeta)^{\sigma}}{\Gamma(1+\sigma)} \\
+2\left(\hbar^{2}+\hbar^{3}\right) \frac{(k \zeta)^{2 \sigma}}{\Gamma(1+2 \sigma)}-\hbar^{3} \frac{(k \zeta)^{3 \sigma}}{\Gamma(1+3 \sigma)}
\end{array}\right],
\end{aligned}
$$

and so on. Finally, the non-differentiable solution $\omega$ of (41), is

$$
\omega(\eta, \mu, \zeta)=(\sqrt{\eta \mu})^{\sigma}\left[\begin{array}{c}
1+(-\hbar)\left(3+3 \hbar+\hbar^{2}\right) \frac{(k \zeta)^{\sigma}}{\Gamma(1+\sigma)}+ \\
+\hbar^{2}(3+2 \hbar) \frac{(k \zeta)^{2 \sigma}}{\Gamma(1+2 \sigma)}-\hbar^{3} \frac{(k \zeta)^{3 \sigma}}{\Gamma(1+3 \sigma)}+\cdots
\end{array}\right] .
$$

By replacing $\hbar=-1$ in (53), we get

$$
\omega(\eta, \mu, \zeta)=(\sqrt{\eta \mu})^{\sigma}\left[1+\frac{(k \zeta)^{\sigma}}{\Gamma(1+\sigma)}+\frac{(k \zeta)^{2 \sigma}}{\Gamma(1+2 \sigma)}+\frac{(k \zeta)^{3 \sigma}}{\Gamma(1+3 \sigma)}+\cdots\right]
$$

and

$$
\omega(\eta, \mu, \zeta)=(\sqrt{\eta \mu})^{\sigma} E_{\sigma}(k \zeta)^{\sigma} .
$$

Note that, in the case $\sigma=1$, we obtain the same solution obtained in [33] by homotopy perturbation method and by conformable reduced differential transform method in [30].

\section{Conclusions}

In our study, the LFHAM method was applied successfully to local fractional biological population models and the results have been compared with integer order in [30]. The use of the LFHAM method is used to obtain the non-differentiable solution of nonlinear biological population models on Cantor sets. It was shown that this method is powerful and effective because it enabled us to solve these two nonlinear local fractional partial differential equations. By this method, the solution is given in the form of a series that converges quickly to the exact solution if it exists. This is confirmed by the results obtained after the application of this method to the two examples proposed in this work. Therefore, it can be said that the LFHAM can be applied to solve other nonlinear partial differential equations with local fractional derivative.

Author Contributions: Writing-original draft preparation, D.Z., M.H.C.; writing-review and editing, M.H.C., D.B.; validation, D.B., K.B.; supervision, K.B. All authors have read and agreed to the published version of the manuscript.

Funding: This research received no external funding.

Conflicts of Interest: The authors declare no conflict of interest. 


\section{References}

1. Cattani, C. A review on Harmonic Wavelets and their fractional extension. J. Adv. Eng. Comput. 2018, 2, 224-238. [CrossRef]

2. Yang, A.M.; Zhang, Y.Z.; Cattani, C.; Xie, G.N.; Rashidi, M.M.; Zhou, Y.J.; Yang, X.J. Application of local fractional series expansion method to solve Klein-Gordon equations on Cantor sets. Abstr. Appl. Anal. 2014, 2014, 372741. [CrossRef]

3. Yoku, A.; Gülbahar, S. Numerical Solutions with Linearization Techniques of the Fractional Harry Dym Equation. Appl. Math. Nonlinear Sci. 2019, 4, 35-42. [CrossRef]

4. Al-Ghafri, K.S.; Rezazadeh, H. Solitons and other solutions of $(3+1)$-dimensional space-time fractional modified KdV-Zakharov-Kuznetsov equation. Appl. Math. Nonlinear Sci. 2019, 4, 289-304. [CrossRef]

5. Khan, M.A.; Hammouch, Z.; Baleanu, D. Modeling the dynamics of hepatitis E via the Caputo-Fabrizio derivative. Math. Model. Nat. Phenom. 2019, 14,311. [CrossRef]

6. Gao, W.; Ghanbari, B.; Baskonus, H.M. New numerical simulations for some real world problems with Atangana-Baleanu fractional derivative. Chaos Solitons Fractals 2019, 128, 34-43. [CrossRef]

7. Gao, W.; Ismael, H.F.; Mohammed, S.A.; Baskonus, H.M.; Bulut, H. Complex and real optical soliton properties of the paraxial nonlinear Schrödinger equation in Kerr media with M-fractional. Front. Phys. 2019. [CrossRef]

8. Gao, W.; Veeresha, P.; Prakasha, D.G.; Baskonus, H.M.; Yel, G. A powerful approach for fractional Drinfeld-Sokolov-Wilson equation with Mittag-Leffler law. Alex. Eng. J. 2019, 58, 1301-1311. [CrossRef]

9. Alvaro, H.S.; Lorenzo, J.M.H. Reaction-Diffusion Equations: A Chemical Application. Sci. Tech. 2010, 46, 134-137.

10. Haff, P.K. Grain flow as a fluid-mechanical phenomenon. J. Fluid Mech. 1983, 134, 401-430. [CrossRef]

11. Radinschi, I.; Covatariu, G.; Cazacu, M.M. Maple Program for Studying Physics Phenomena with Applications in Civil Engineering. Intersections Intersecţii 2016, 13, 107-118.

12. Rìos, E.; Stern, M.D. Calcium in Close Quarters: Microdomain Feedback in Excitation-Contraction Coupling and Other Cell Biological Phenomena. Annu. Rev. Biophys. Biomol. Struct. 1997, 26, 47-82. [CrossRef] [PubMed]

13. Al-Mazmumy, M.; Al-Malki, H. The Modified Adomian Decomposition Method for Solving Nonlinear Coupled Burger's Equations. Abs. Appl. Anal. 2012, 2012, 727031. [CrossRef]

14. Adomian, G.; Rach, R. Equality of partial solutions in the decomposition method for linear or nonlinear partial differential equations. Comput. Math. Appl. 1990, 10, 9-12. [CrossRef]

15. He, J.H. Homotopy perturbation technique. Comput. Methods Appl. Mech. Eng. 1999, 178, 257-262. [CrossRef]

16. He, J.H. A new approach to nonlinear partial differential equations. Commun. Nonlinear Sci. Numer. Simul. 1997, 2, 203-205. [CrossRef]

17. Daftardar-Gejji, V.; Jafari, H. An iterative method for solving nonlinear functional equations. J. Math. Anal. Appl. 2006, 316, 753-763. [CrossRef]

18. Liao, S.J. The Proposed Homotopy Analysis Technique for the Solution of Nonlinear Problems. Ph.D. Thesis, Shanghai Jiao Tong University, Shanghai, China, 1992.

19. Liao, S.J. Beyond Perturbation: Introduction to Homotopy Analysis Method; Chapman and Hall/CRC Press: Boca Raton, FL, USA, 2003.

20. Liao, S.J. On the homotopy analysis method for nonlinear problems. Appl. Math. Comput. 2004, 147, 499-513. [CrossRef]

21. Liao, S.J. Notes on the homotopy analysis method: Some definitions and theorems. Commun. Nonlinear Sci. Numer. Simul. 2009, 14, 983-997. [CrossRef]

22. Ayub, M.; Rasheed, A.; Hayat, T. Exact flow of a third grade fluid past a porous plate using homotopy analysis method. Int. J. Eng. Sci. 2003, 41, 2091-2103. [CrossRef]

23. Abbasbandy, S. The application of homotopy analysis method to nonlinear equations arising in heat transfer. Phys. Lett. A 2006, 360, 109-113. [CrossRef]

24. Abbasa, Z.; Vahdatia, S.; Ismaila, F.; Karimi Dizicheha, A. Application of Homotopy Analysis Method for Linear Integro-Differential Equations. Int. Math. Forum 2010, 5, 237-249.

25. Hashim, I.; Abdulaziz, O.; Momani, S. Homotopy analysis method for fractional IVPs. Commun. Nonlinear Sci. Numer. Simul. 2009, 14, 674-684. [CrossRef] 
26. Odibat, Z.; Momani, S.; Xu, H. A reliable algorithm of homotopy analysis method for solving nonlinear fractional differential equations. Appl. Math. Model. 2010, 34, 593-600. [CrossRef]

27. Zhang, Y.; Cattani, C.; Yang, X.J. Local fractional homotopy perturbation method for solving non-homogeneous heat conduction equations in fractal domains. Entropy 2015, 17, 6753-6764. [CrossRef]

28. Maitama, S.; Zhao, W. Local fractional homotopy analysis method for solving non-differentiable problems on Cantor sets. Adv. Diff. Equ. 2019, 127, 2-22. [CrossRef]

29. Mohyud-Din, S.T.; Ali, A.; Bin-Mohsin, B. On biological population model of fractional order. Int. J. Biomath. 2016, 9, 1650070. [CrossRef]

30. Acan, O.; Mohamed Al Qurashib, M.; Baleanu, D. New exact solution of generalized biological population model. J. Nonlinear Sci. Appl. 2017, 10, 3916-3929. [CrossRef]

31. Yang, X.J. Fractional Functional Analysis and Its Applications; Asian Academic: Hong Kong, China, 2011.

32. Yang, X.J. Local Fractional Calculus and Its Applications; World Science Publisher: New York, NY, USA, 2012.

33. Roul, P. Application of Homotopy Perturbation Method to Biological Population Model. Appl. Appl. Math. 2010, 5, 272-281.

(C) 2020 by the authors. Licensee MDPI, Basel, Switzerland. This article is an open access article distributed under the terms and conditions of the Creative Commons Attribution (CC BY) license (http://creativecommons.org/licenses/by/4.0/). 